\section{Christian Schopper}

\section{Einleitung}

In den letzten Jahren haben sich die Betrachtung und auch die Behandlung in der Onkologie grundlegend gewandelt. Es wurde eine Vielzahl neuer therapeutischer Optionen entwickelt, die auf der Basis des Verständnisses der Krebserkrankung als einer Immunerkrankung - präziser gesagt: Autoimmunerkrankung - beruhen. Im erweiterten anthroposophischen Sinne bedeutet das, dass es sich um eine Erkrankung handelt, die zutiefst dass ICH bzw. den ganzen Menschen anspricht. Insofern ist die Anschauung längst verlassen worden, dass die Krebserkrankung eine lokale Zellerkrankung ist und auch entsprechend nur lokal durch Operation bzw. Bestrahlung oder Zytostatika, die regional den Krebs zerstören, zu behandeln ist. So finden sich zudem in den vielfältigen Ergebnissen der Forschung zur Psychogenese der Krebserkrankung Einflüsse durch psychosoziale und biografische Faktoren, insbesondere auch bei Spontanremissionen.

Es gibt mittlerweile eine spezielle eigene Zeitschrift, das internationale Journal Psychooncology, das sich ausschliesslich mit psychoonkologischer Forschung beschäftigt. Sie hat einen soliden Impact-Faktor; daneben finden sich immer wieder wesentliche Artikel in zunehmendem Umfang und in steigender Qualität in den internationalen Journalen für Psychosomatik (Psychosomatics usw.), aber auch im Rahmen der Traumaliteratur (bezüglich «posttraumatic growth» und «risk factors» usw.).

Es kann nicht Aufgabe dieses Übersichtsartikels sein, spezifische Frage-

\title{
Anthroposophische Psychoonkologie
}

stellungen bzw. Reviews in der internationalen psychoonkologischen Literatur darzustellen und aufzuarbeiten.

Die Anthroposophische Krebstherapie, die sich vor allem auf die Forschungen von Rudolf Steiner bezüglich der Mistel als zentrales Krebsheilmittel für den Menschen gründet, behandelt seit ihren Anfängen im Jahr 1917 immer den ganzen Menschen, d.h. seinen Leib, seine Seele und seinen Geist. Aus einer anthroposophisch erweiterten Psychotherapie und integrativen Psychosomatik ergeben sich vielfältige Ansätze einer anthroposophischen Psychoonkologie. Diese wird in einigen Grundzügen im vorliegenden Artikel dargestellt und in den Kontext der Ausführungen Rudolf Steiners über die Krebskrankheit und der wesentlichen Referenzautoren einer modernen Psychoonkologie gestellt.

\section{Das Bild der Krebserkrankung in der Anthroposophischen Medizin}

«In der Mistel ist die wirksame Natur irrsinnig geworden, sie macht alles zur Unzeit ... das ist gerade dasjenige, was man (...) benützen muss, wenn auf der anderen Seite der menschliche Organismus physisch irrsinnig wird, und das wird er ja zum Beispiel gerade in der Karzinombildung» (Vortrag von Rudolf Steiner, gehalten am 2. April 1920) [1].

Rudolf Steiner, der Begründer der Anthroposophischen Medizin, gab vielfache Darstellungen vor Ärzten über ein tieferes Verständnis der Krebserkrankung und insbesondere
Hinweise auf Behandlungsmöglichkeiten, die in der damaligen Zeit der Medizin erstmalig pharmakologische Empfehlungen beinhalteten [1-3]. Dabei pflegte er eine wissenschaftliche Zusammenarbeit mit Dr. med. Ita Wegman, die 1917 in Zürich erstmals ein Mistelpräparat anwendete, und verschiedenen Ärzten in seinem Umfeld. Bereits nach dem Ersten Weltkrieg wurde die Weleda in Arlesheim (und später im deutschen Schwäbisch Gmünd) begründet, die sich zu einem internationalen Heilmittel-Konzern entwickelt hat und neben anderen anthroposophischen Heilmittelherstellern bis heute die Mistelpräparate als einen zentralen Pfeiler ihres Unternehmenskonzepts anbietet. $\mathrm{Zu}$ einer Zeit, als der Begriff des Immunsystems noch nicht beschrieben war, erst ganz ansatzweise überhaupt etwas über die Hormonregulation bekannt und praktisch noch keine detaillierte Molekularbiologie der Zellen und genaue $\mathrm{Pa}$ thogenese der Erkrankungen vorhanden war, hat Rudolf Steiner durch seine Methode der Geistesforschung bahnbrechende Erkenntnisse über die Krebserkrankung bzw. den krebserkrankten Menschen entwickelt und viele der heutigen Anschauungen vorweggenommen. In den Darstellungen Rudolf Steiners ist die Krebserkrankung immer eine Erkrankung des ganzen Menschen, die vor allem in einer Dysregulation der Lebenskräfte besteht, die im sogenannten Ätherleib zu suchen ist, und wenn das ICH einen Mangel der Kontrollmechanismen aufweist. Es gilt, die aus dem körpereigenen Lebenskräftestrom herausgefallenen wuchernden Krebszellen in den körpereigenen Lebensleib einzu-

\section{KARGER}

(c) 2017 S. Karger GmbH, Freiburg

Fax +497614520714
Dr. med. Christian Schopper, MHBA

Arzt für Neurologie/Psychiatrie/Psychotherapie FMH \& anthroposophische Medizin VAOAS/GAÄD

Praxis Vogelsang

Vogelsangstrasse 52, 8006 Zürich, Schweiz

praxis.vogelsang@bluewin.ch 
binden und dem ICH wieder die Verfügungsgewalt über die Wärme bzw. gestörten Wärmeprozesse zu geben, da die seelisch-leibliche Eigenwärme als der zentrale Vermittler der ICHWirkungen im Leib angesehen wird. Viele Krebspatienten leiden unter Wärmestörungen, haben jahre- bis jahrzehntelang eine «Anergie», d.h. fehlende Infektionen in der Anamnese, und reagieren nicht mehr mit fieberhaften Erkrankungen. Das Immunsystem ist erstarrt; es gibt praktisch keine Wärmeregulation mehr. Rudolf Steiner entwickelte damals in eigenen Worten den Begriff der Selbstregulation und der Selbstheilungskräfte $[4,5]$, oder wie Paracelsus es ausspricht, der «inwendige Arzt» als der eigentliche Heiler, was bedeutet, dass die Lebenskräfte als zentrale regulatorische Instanz für Heilung und Genesung bei jeder Krankheit wirken.

Die Misteltherapie greift nun tief in den Wärmeorganismus ein, aktiviert Lebensprozesse und versucht diese so zu stärken, dass das Tumorgeschehen wieder eingegliedert wird. Zudem hat die Mistel zytotoxische und immunmodulatorische Wirkungen, die heute detailliert in der modernen Onkologie und Phytotherapie-Grundlagenforschung gezeigt werden können.

Immer jedoch ist die Krebserkrankung eine zentrale Herausforderung im Sinne der Generierung existenzieller Ängste und stellt zumeist einen bedrohlichen Einschnitt in die gesamte Biografie des Betroffenen dar.

\section{Zur allgemeinen Psychoonkologie}

Kaum eine Erkrankung generiert derartige Ängste bei den Patienten und schafft ausgeprägte Hilflosigkeit, Ohnmachtserleben und Existenzängste. Dies äussert sich auch oft in einer geradezu devoten, unterwürfigen Auslieferung an die Onkologie, die mit Zahlen der Überlebensraten operiert sowie oftmals vom Patienten Auslieferung und Unterwerfung unter die Therapieschemata der Onkologie fordert und dabei Heilung und Therapierbarkeit suggeriert. Das führt zu einer erheblichen Abhängigkeit und Dominanzbeziehung, was auch zur Folge haben kann, dass immer wieder Patienten vollständig aus der schulmedizinischen Onkologie aussteigen und zum Teil obskure alternativmedizinische Wege wählen. Zudem erleben sie sich durch den Prozess der Diagnosestellung und Therapieberatung durch den Onkologen erheblich traumatisiert. Es wird somit von der «klassischen Onkologie» häufig mit der Angst gegenüber der Krankheit gewirkt.

Dagegen haben sich in den letzten Jahren enorm hilfreiche Therapieansätze entwickelt, welche die Selbstheilungskräfte, das "psychische Immunsystem» sowie die Resilienz der Betroffenen im Sinne tiefergehender salutogenetischer Selbstheilungsaktivierung unterstützen. Es gibt zwar nicht DIE Krebspsyche bzw. keine tumorspezifische Krebspsyche, jedoch gibt es eindeutige Profile, die psychosomatisch bei Tumorpatienten evaluiert werden können und erfolgreich in eine begleitende psychoonkologische Behandlung integrierbar sind.

Ich möchte hier stellvertretend drei Autoren und ihre Ansätze nennen, die zu den Pionieren der Psychoonkologie gehören:

(1) Lawrence LeShan $[6,7]$ hat in vielfältigen Studien die Wirksamkeit von Einzel- und insbesondere Gruppenpsychotherapien im Verlauf von Krebserkrankungen zeigen können. Er geht dabei methodisch von einem tiefenpsychologisch-humanistischen Modell des Menschen aus. LeShan war früher ausschliesslich ein somatisch denkender Onkologe und hatte dann bemerkt, dass er erfolgreicher arbeiten kann, wenn er den ganzen Menschen therapiert und die Psyche und Biografie des Patienten im Sinne der modernen Tiefenpsychologie und humanistischen Psychologie mit einbezieht. Dies hat er in grossartigen Büchern und auch vielen Aufsatz-Publikationen dargestellt.

(2) Bernie Siegel $[8,9]$ war ebenso ein ausschliesslich somatisch denkender Onkologe und merkte, dass für ihn als zentrale Instanz der Therapie die "positive Psychologie», die Selbstheilungsaspekte und die religiös-spirituelle Dimension in der Krankheitsbewältigung wesentlich wurden. In seinen Schriften, Publikationen und Büchern hat er Konzepte entwickelt, den Patienten Mut zu machen sowie gezielt mit inneren Dimensionen des Selbst und transzendenten Dimensionen zu arbeiten. Damit war der Patient auch mitbeteiligt am Verständnis von Prognose und Outcome. Es ist heute üblich, dass die Outcome-Zahlen von Patienten ausschliesslich im Hinblick auf die statistisch relevante «State of the Art» der krankheitsbildtypologischen onkologischen Therapien gesehen werden. Die Ergebnisse der Psychoonkologie, aber auch die allgemeinen klinischen Erfahrungen vieler Kollegen sowie meine eigenen, seit 30 Jahren in der psychosomatischen Praxis erworbenen Erfahrungen widersprechen dem deutlich.

(3) Carl Simonton war ein österreichischer, in den USA lebender und wirkender Onkologe, der in seinen Büchern (z.B. [10]) und vielfältigen Kursen ein lehrbares Konzept entwickelt hat, wie der von Krebs betroffene Patient aktiv an seiner Heilung und der Bewältigung von Angst, insbesondere Todesangst, arbeiten und mitgestalten kann. Sein Konzept ist spirituell orientiert und integriert die transzendente transpersonale Dimension. Der Patient wird als Mitbeteiligter und aktiver Mitgestalter seines Schicksals gesehen, ganz im Sinne von Viktor Frankl [11] und seiner Existenzanalyse und Logotherapie. Dabei hat Simonton Programme mit Imaginati- 
Abb. 1. Krebsärzte würden selbst niemals einer Chemotherapie zustimmen (Studie des Hutchinson Krebsforschungsinstituts in 2012; modifiziert nach: $h$ ttp://faq4h.bplaced.net).

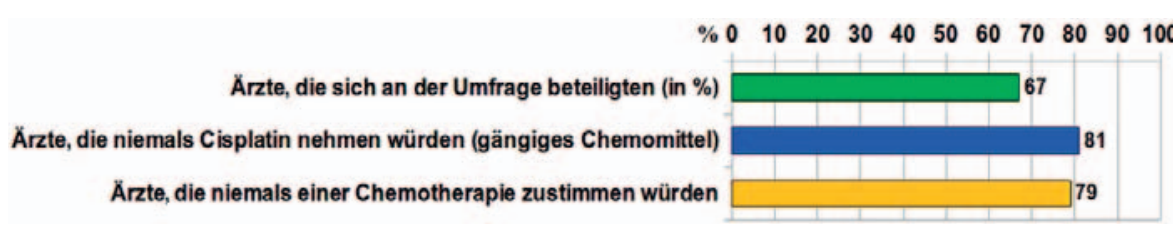

onsübungen, Meditationsübungen, Übungen zur Stärkung von Selbstwertgefühl, ICH-Stärkung und anderes entwickelt. Diese sind anthroposophisch gut verständlich und übersetzbar in die Menschenkunde der Lebenskräfte und des ICH. Stellvertretend für dieses Verständnis sei hier Josef Ulrich erwähnt, langjähriger Leiter der Abteilung für Kunsttherapie in der anthroposophischen Krebsklinik Öschelbronn bei Pforzheim, Simonton-Therapeut und anthroposophischer Psychoonkologe. In seinem Buch «Selbstheilungskräfte» [12] entwickelt er das integrative Behandlungsmodell, das auf Simontons Forschungen und auf der anthroposophisch-integrativen, den Anregungen Rudolf Steiners folgenden Psychoonkologie basiert - sowohl in der Form einer Gruppentherapie als auch mittels eines Konzepts für den einzelnen Patienten mit individueller Beratung und Kunsttherapie.

Onkologen selbst scheinen nicht besonders viel Vertrauen in ihre Therapien zu haben. Abbildung 1 zeigt in erschütternder Weise auf, dass sie selbst meist keiner Chemotherapie zustimmen würden.

Eines der zentralsten Forschungsergebnisse der Psychotherapieforschung der letzten Jahrzehnte, das vielfach repliziert ist, zeigt die Wichtigkeit der Arzt-Patienten-Beziehung in allen Fachgebieten, nicht nur in der Psychotherapie. Wirkungen und Nebenwirkungen von Medikamenten, Nocebo und Placebo sind immer innerhalb der jeweiligen Beziehung zu sehen, welche die primären frühen Bindungserfahrungen wiederholen; hier möchte ich auf wesentliche For- schungsergebnisse von John Bowlby und Mary Ainsworth $[13,14]$ hinweisen, die im deutschen Sprachraum unter anderem durch Klaus und Karin Grossmann [15] bekannt wurden. So hängt z.B. der Opiatverbrauch in einer Nachtschicht nur bedingt von den objektiven Krankheitsbildern, sondern wesentlich von Persönlichkeit, Psyche und Verhalten oder auch Bindungsund Beziehungsfähigkeit der Nachtwache zu den entsprechenden Schmerzpatienten bzw. onkologischen Patienten ab.

Psychoonkologie möchte zum einen die Dimension der reflektierten Beziehung in die onkologische Therapie einbeziehen und zum anderen die biografische Relevanz und das Auftreten der Krebserkrankung verstehen und entsprechend bearbeiten.

\section{Anthroposophische integrative Psychosomatik und Psychotherapie}

Ein wesentliches Element bildet die sogenannte Biografie-Arbeit [16], d.h. das Anschauen der Biografie, wie sie sich als Entwicklung über Sieben-Jahres-Perioden gestaltet. Das Auftreten der Erkrankung in einem spezifischen Alter mit den entsprechenden Entwicklungsaufgaben des jahrsiebtspezifischen Alters hat hier eine grosse Bedeutung und wird in einer entsprechend darauf fokussierten Psychotherapie mit dem Patienten erarbeitet.

Dabei ist die im Rahmen der von Rudolf Steiner entwickelten Menschenkunde, d.h. einer wissenschaftlichen, phänomenologischen, beschreibbaren, lehr- und verstehbaren Betrachtung von Leib, Seele und Geist, insbesondere der sogenannten Lebenskräfte, des «Ätherleibes», vor allem die Betrachtung der Wärmeprozesse in der Biografie im geistigen, seelischen und leiblichen Bereich zentral. Dies stellt auch einen wesentlichen Fokus jeder anthroposophischen integrativen Psychosomatik und Psychotherapie dar.

Grundlegend ist die Arbeit an der Angst bzw. die konstruktive, integrierende Auseinandersetzung mit Sterben und Tod. Viele Patienten haben keinerlei Beziehung zum GöttlichGeistigen mehr, auch nicht zu Spiritualität und Meditation. Häufig ist ihnen jede transpersonale Ebene verlorengegangen. Und falls diese vorhanden ist, wird sie häufig durch die Wucht der Krebserkrankung und die «niederschmetternde» Prognose der Onkologie mit Überlebenszeiten, die vorgesetzt werden wie unausweichliche Fahrpläne, ausgelöscht und ausser Kraft gesetzt. Für den Patienten ist es notwendig, dass er wieder ein tieferes Vertrauen in seinen Körper und seine Seele findet und dabei in irgendeiner Form Zugang zu inneren Kraftquellen wie Gebet, Meditation und Achtsamkeitsübungen erhält. Auch müsste für ihn erlebbar werden, dass er selbst an seiner Genesung beteiligt ist. Das bedeutet das Auffinden des Persönlichkeitszentrums, seiner ICH-Instanz, des Persönlichkeitskerns, der in der Krankheitsbewältigung und in jeder Therapie begleitend mittätig ist. Praktisch bedeutet es, durch Zusatztherapien genau diese Instanzen zu stärken und zu fördern. Zudem sollte, da häufig die onkologischen Therapien die Lebenskräfte massiv auszehren, an intensiver Ressourcenaktivierung im Hinblick auf die Lebenskräfte gearbeitet werden. So konnte in einem Cross- 
over-Design gezeigt werden, dass deutlich geringere Nebenwirkungen bei höherer Ansprechrate bei Zytostatika-Infusionen $\mathrm{zu}$ verzeichnen sind, wenn die Patienten gleichzeitig Naturund Tierfilme ruhig und achtsam anschauen. Die Aktivierung zentraler $\mathrm{ICH}$-Ressourcen ist hier vorrangig als "psychosomatische Wärmetherapie» $\mathrm{zu}$ nennen. Weiter braucht der Krebspatient unbedingt eine stabile, vertrauensvolle Begleitung, die personalisiert sein muss. Hier verweise ich auf die wichtige Publikation von Gerd Nagel [17], der selber als Ordinarius für biologische Onkologie und Ärztlicher Direktor der Klinik für Tumorbiologie in Freiburg im Breisgau an einer Leukämie erkrankt war und alle seine dann vorgeschlagenen psychoonkologischen bzw. therapeutischen Elemente einer «Patientenkompetenz» an sich selbst erfahren und erforscht hat. Sein Ansatz der Patientenkompetenz besteht in der Integration von Elementen der anthroposophischen Biografie-Arbeit bzw. der Misteltherapie mit der vorbehaltslosen Analyse der Krebserkrankung im Hinblick auf Biografie und Persönlichkeit - in Form einer detaillierten und schonungslosen Analyse der Therapieziele und des Gesundungswillens. Eine integrative anthroposophische Psychoonkologie versucht, mit dem Patienten intensiv am Gesundungswillen, der Zukunftsorientierung und den Zukunftsressourcen zu arbeiten. Nagel hat diesbezüglich wesentliche Elemente erarbeitet.

Der Aspekt der biografischen Analyse und Einbettung der Erkrankung ist meist damit verbunden, dass die Diagnose der Krebserkrankung wie aus «heiterem Himmel» kommt, häufig ohne Vorboten auftaucht, ohne Frühsymptome gestellt wird und wie ein «Donnerschlag» im Leben wirkt. Die Betroffenen werden in einem Moment aus Beruf, Partnerschaft und Leistungsbezogenheit herausgeworfen sowie plötzlich mit dem Tod, infausten Überlebensprognosen und anderem Unvorstellbarem konfrontiert.
Häufig wird mit massivem Zeitdruck seitens der Onkologie gearbeitet, d.h. chirurgische Interventionen, biologische Krebstherapie mit Antikörpern und Zytostatika werden den Patienten unter grossem Zeitdruck vorgesetzt. Im Rahmen der bekannten Phasen der Krankheitsverarbeitung (siehe die entsprechenden psychosomatischen Forschungen, z.B. [18]) bleibt den Patienten in der Regel keine Zeit, Diagnose und Krankheit überhaupt zu verarbeiten, geschweige denn eine Einbettung in die Biografie vorzunehmen. Selbst basales Coping findet häufig nicht statt. Dieses muss dann dringend in einer entsprechenden biografischen Arbeit nachgeholt werden. Zentral ist hier der Aspekt zu bearbeiten, was die Lebensziele und Lebensmotive anbetrifft. Der Krebspatient braucht dringend weitere Lebensziele und Zukunftsperspektiven. Diese müssen in der Regel in der Therapie gemeinsam erarbeitet werden. Häufig sind viele Patienten wie aus ihrer Biografie «herausgefallen», stellvertretend seien einige schriftstellerische Bilder der Krebserkrankung und ihrer zwangsläufigen zum Tode führenden Signatur aufgeführt: «Mars» von Fritz Zorn [19], der schliesslich alle Therapien aufgegeben und beendet und keinerlei Zukunftsvisionen oder -perspektive für sein Leben mehr hatte.

Des Weiteren sei auf das Buch «Die Wand» von Marlen Haushofer [20] hingewiesen, ein prämiertes Buch der berühmten österreichischen Schriftstellerin, die bei Fertigstellung des Buches bereits an Brustkrebs erkrankt war und bald darauf starb. Man kann das Buch als die Pathografie einer psychoonkologischen Sichtweise der Brustkrebserkrankung lesen. Häufig schildern Frauen mit einer Brustkrebserkrankung, wie in diesem Buch, dass sie den Bezug zur Umwelt verloren haben, wie in einem inneren Gefängnis leben, keine wirkliche Beziehung zu Beruf, Partner und Kindern mehr haben und sich wie abgekapselt und ausgegrenzt vom Leben fühlen sowie keinen inneren Zugriff mehr zur
Seele, zu Visionen und zur Zukunftsorientierung haben. Das zeigt dann auch genau die entsprechenden Aspekte, die in einer psychoonkologischen integrativen Therapie erarbeitet werden sollten und müssten. Die bewusste Auseinandersetzung mit der Biografie, insbesondere den Beziehungen, ist dabei ein zentraler Baustein.

Tiziano Terzani, der berühmte Journalist, schreibt in seinem Bestseller "Noch eine Runde auf dem Karussell» [21] schonungslos über die Entstehung und den Verlauf seiner Krebserkrankung, aber auch seine Entscheidung, diese zuzulassen und in aller Ruhe, "wie ein Brahmane, zu gehen». An seinem Beispiel wird die viel diskutierte «Trauma-Genese» der Krebserkrankung sehr sichtbar, die hier nur angedeutet werden soll. Zumindest traumatische Lebensereignisse spielen in der Forschungsliteratur, vor allem auch in den Patientenschilderungen und deren Selbstbildern, wie urbildhaft bei Terziano Terzani, eine grosse Rolle.

So ist die Frage des Selbstbildes, des inneren Selbstverständnisses, von zentraler Bedeutung. Insbesondere Brustkrebs-Patientinnen sind im Interaktionsstil häufig eher angepasst, wobei die Erfüllung der Bedürfnisse anderer Menschen und Altruismus unter Vernachlässigung jeder Selbstfürsorge für sie das Wichtigste sind. Die Bedürfnisse des Anderen werden über die eigenen gestellt, wobei letztere wie abgespaltet werden. Die für die Therapie zentral wichtige Fähigkeit zur Selbstfürsorge ist vielen Patienten völlig unbekannt und muss intensiv in der ambulanten oder stationären Psychotherapie erarbeitet werden. In den von mir langjährig geleiteten psychosomatischen Kliniken mit der Ausrichtung einer anthroposophischen integrativen Psychosomatik stellte sich die intensive Bearbeitung der Selbstfürsorge als zentralster Aspekt der Genesung und der Heilung dar. Simonton hob hervor, dass jede Krebserkrankung zu jedem Zeitpunkt heil- 
bar ist, und machte es zur Grundlage seiner Seminare, auch bei Patienten auf Palliativstationen. Der Betroffene kann die Erkrankung immer überwinden, nur ist manchmal der Leib schon zu mitgenommen und ausgezehrt, sodass es der Mensch nicht immer schafft, die Lebenskräfte weiter zu tragen. Dies ist eine vollkommen andere Perspektive, als es sonst in der Onkologie üblich ist.

Eine intensive Psychotherapie der Selbstfürsorge setzt einen tiefen Selbstbewusstseins- und Selbsterkenntnisprozess bezüglich des Leibes voraus mit dem Ziel, dass die Betroffenen ihren Leib als lebendig und beseelt erleben können. Hier spielen insbesondere die anthroposophischen Leibtherapien eine wesentliche und vielfach erfolgreiche Rolle, z.B.:

- rhythmische Einreibungen nach Wegman und Hauschka, Teil- und Ganzkörpereinreibungen,

- die rhythmische Massage nach Wegman und Hauschka, als zentrale Regulationstherapie vegetativer Sympathikus-Übersteuerung,

- das Öldispersionsbad nach Junge und

- die kraniosakrale Osteopathie als zentrale Regulationstherapie der Lebenskräfte.

Im Rahmen eines besseren Körperbewusstseins, genauer gesagt Leibbewusstseins, wird den Patienten ein anderer Umgang und eine neue Selbstfürsorglichkeit mit ihren Leibesprozessen möglich. Hier spielen auch sensorische Naturerfahrungen mit intensiven Natur- oder auch Kunsterlebnissen eine grosse Rolle. Nagel beschreibt, wie er nach der Diagnose der Leukämie feststellte und völlig erschüttert darüber war, dass er als Naturliebhaber keinerlei Wahrnehmung und sensorische Erlebniskraft mehr von der Natur und dabei insbesondere von Bäumen hatte [17]. Die Genesung war dann für ihn da, als dies wieder möglich war, selbstverständlich integriert in die «State-of-the-Art» Leukämietherapie, die er bei Kollegen in London durchführen liess. Wie aus den Beispielen ersichtlich, geht es in der Anthroposophischen Medizin und Psychoonkologie nicht um eine komplementäre, sondern um die integrative Behandlung.

Die Selbstfürsorge im seelischen Bereich dreht sich um die Frage der eigentlichen seelischen Bedürfnisse. Abgrenzung und Autonomie sind häufig Fremdworte insbesondere für Menschen, die in abhängigen Beziehungen leben, sich unterwürfig zeigen und es jedem recht machen wollen. Das handelnde ICH, die selbstbewusste Seele hat sich dabei zurückgezogen. In der Auseinandersetzung mit der Krebserkrankung müssen seelische Abgrenzungsqualitäten und die ICHStärkung sowie die unten aufgeführten sieben Hauptfaktoren der Resilienz erübt werden. Nagel et al. [17] nennen dies Patientenkompetenz; Grossarth-Maticek [4, 5] konnte das von ihm entwickelte «Autonomietraining» klinisch erfolgreich durchführen und mit statistisch signifikanten Ergebnissen publizieren.

\section{Die sieben Hauptfaktoren der Resilienz}

\section{- Akzeptanz}

- Lösungsorientierung

- Opferrolle verlassen

- Verantwortung übernehmen

- Selbstwirksamkeit

- Netzwerk-Orientierung

- Zukunftsplanung

Insbesondere bei der Gruppenpsychotherapie spielt es eine grosse Rolle, dass der Patient sich im wohlwollenden Spiegel der anderen wieder erfahren und neu sehen lernen kann. Verhaltensänderungen, zum eigenen Ich und zu seiner Biografie stehen können, sind dann ganz neue und zentrale «salutogenetische» Erfahrungen.

Das «nicht gelebte Leben» ist eine zentrale, zu therapierende Dimension jeder psychoonkologischen Arbeit und biografischen Analyse. Auf der ICH-Ebene bedeutet Selbstfürsorge, den zumeist unterdrückten und nicht ausgelebten Impulsen von Autonomie, wirklichen Visionen und Interessen zu folgen, diese zu erarbeiten und im Alltag umzusetzen. Dies bedeutet, die eigene Biografie als ICH-gesteuert und ICH-geführt zu erleben und nicht in der «Zahnradbahn des Lebens» zu sitzen. Den Roman «Nachtzug nach Lissabon» von Pascal Mercier [22] könnte man auch als Metapher für eine quasi psychoonkologische Reifung mit biografischer Neukonstruktion interpretieren, wo die Hauptperson, bevor sie dann die infauste Diagnose einer konsumierenden Erkrankung erhält, diese dann ganz ruhig annehmen kann, da sie ihre Biografie im Hinblick auf Autonomie und Selbstfürsorge mittlerweile neu konstruieren konnte, "gelebt hat» und in wenigen Wochen das zuvor «nicht gelebte Leben» nachholt. Diese biografische Neukonstruktion ist wohl der zentrale Ort wie auch der prognostisch wirksame Bestandteil der Therapie bzw. Heilung, welche die anthroposophische Psychoonkologie der rein somatischen Orientierung hinzufügen kann.

Im Blick auf den erweiterten Wärmebegriff bedeutet es auch, dass Patienten ganz neu für sich im Sinne tieferer Selbstfürsorge den Begriff des «Liebevollen» erfahren und erleben und an sich selbst erarbeiten. Die liebevolle Beziehung zu sich selbst war zumeist weitgehend verlorengegangen, und die Liebe als zentrale, evolutionäre und transpersonale Kraft ist häufig nicht mehr erfahrbar gewesen. Auch dies ist ein zentraler Fokus im Hinblick auf die Integration transpersonaler Dimensionen bzw. auf die ICH-ausgerichtete, anthroposophische Psychoonkologie. Immer wieder berichten Patienten tief berührt von transpersonalen Erlebnissen mit ihrem Engel, von helfenden Verstorbenen, von Christuserlebnissen usw., wenn sie sich einmal auf einen derartigen Prozess eingelassen haben und im behandelnden Arzt einen entsprechend «offenen» Zuhörer haben. Auch Schilderungen von Nahtod-Erlebnis- 
sen von Krebspatienten, d.h. Betroffenen, die an der Schwelle zum Jenseits standen und wieder «zurück» kamen, verbunden mit tiefen transpersonalen Erlebnissen, seien hier genannt. Stellvertretend möchte ich zwei Autoren unter transpersonalen Gesichtspunkten nennen: Sabine Mehne in ihren beiden Büchern «Licht ohne Schatten» [23] und «Der grosse Abflug» [24] sowie Anita Moorjani in «Die Geschichte einer Heilung» [25]. Auch können in einer anthroposophischen Palliativmedizin, wie sie z.B. in Berlin in der Klinik Havelhöhe praktiziert und auch gelehrt wird, die oben dargestellten Elemente äusserst hilfreich sein. Mit einer ganzheitlichen, aus der Anthroposophie heraus inspirierten Anschauung kann der Patient dahin begleitet werden, den Tod nicht angstbesetzt als das Ende jeden Lebens zu betrachten, sondern als einen zentralen biografischen Abschnitt und wichtigen Lebensprozess. So hat die anthroposophische Pflege in der onkologischen stationären Therapie viele helfende Massnahmen ausgearbeitet, um den Patient leiblich, seelisch und geistig zu stützen und ihn insbesondere unter dem Aspekt der Wärme zu begleiten.

Auch Elemente des von Rudolf Steiner in vielen Schriften dargestellten seelisch-geistigen Schulungsweges können hilfreich sein. Viele dieser Übungen wirken ICH-Kräfte sowie Seelen- und Lebenskräfte stärkend. Ich verweise an dieser Stelle auf die Ausführungen meines Kollegen Harald Haas [26-28].

Die zentralen Aspekte einer integrativen, anthroposophisch-psychoonkologischen Therapie können dem Patienten unabhängig von Krankheitsstadium, Ausmass der Schmer- zen, Prognose und Überlebenszeiten, Selbstbewusstsein und Selbstsicherheit vermittelt werden und ihn unterstützen, sich wieder an transpersonalen Erfahrungen der belebten Natur, der Engelwelt und anderen Dimensionen des Göttlichen, aber auch an Lebensfreude und intensive Sinnespflege anzuschliessen. Hier kann auch auf das wichtige Buch «Zur Besinnung kommen: Die Weisheit der Sinne und der Sinn der Achtsamkeit in einer aus den Fugen geratenen Welt» von Jon Kabat Zinn [29] verwiesen werden.

Hilde Domin hat in einem grossartigen Gedicht das oben Dargestellte sehr einfach ausgedrückt:

«Nicht müde werden sondern dem Wunder wie einem Vogel die Hand hinhalten» [30].

\section{Literatur}

1 Steiner R: 1. Ärztekurs: Geisteswissenschaft und Medizin, GA 312, ed 7. Dornach, Rudolf Steiner, 1999.

2 Steiner R, Wegman I: Grundlegendes zu einer Erweiterung der Heilkunst, GA 27, ed 8. Dornach, Rudolf Steiner, 2014.

3 Steiner R: Vortrag vom 02.04.1920 aus Heilfaktoren für den sozialen Organismus, GA 198, ed 2. Dornach, Rudolf Steiner, 1984.

4 Grossarth-Maticek R: Selbstregulation, Autonomie und Gesundheit. Krankheitsfaktoren und soziale Gesundheitsressourcen im soziopsycho-biologischen System. Vorworte von Helm Stierlin und Peter Schmidt. Berlin, De Gruyter, 2003.

5 Grossarth-Maticek R: Autonomietraining. Gesundheit und Problemlösung durch Anregung der Selbstregulation. Berlin, De Gruyter, 2000.

6 LeShan L: Psychotherapie gegen den Krebs, ed 10. Stuttgart, Klett Cotta, 2008.

7 LeShan L: Diagnose Krebs: Wendepunkt und Neubeginn, ed 11. Stuttgart, Klett Cotta, 2016.

8 Siegel B: Mit der Seele heilen. Berlin, Econ, 2000.

9 Siegel B: Prognose Hoffnung. Berlin, Ullstein, 2003.

10 Simonton C: Wieder Gesund werden. Reinbek, Rowohlt, 2001.

11 Frankl V: Logotherapie und Existenzanalyse. Texte aus sechs Jahrzehnten. Weinheim, Beltz, 2001.

12 Ulrich J: Selbstheilungskräfte - Quellen der Gesundheit und Lebensqualität. Stuttgart, Freies Geistesleben, 2016.

13 Bowlby J: Über das Wesen der Mutter-KindBindung. Psyche 1959;13:415-456.

14 Ainsworth MD, Blehar M, Waters E, Wall S: Patterns of Attachment: A Psychological Study of the Strange Situation. Hillsdale, NJ, Lawrence Erlbaum, 1978.

15 Grossmann KE, Grossmann K: Bindung und menschliche Entwicklung. John Bowlby, Mary Ainsworth und die Grundlagen der Bindungstheorie. Stuttgart, Klett-Cotta, 2003.

16 Burkhard G: Das Leben in die Hand nehmen Arbeit an der eigenen Biographie. Stuttgart, Freies Geistesleben, 2014.

17 Bopp A, Nagel D, Nagel G: Was kann ich selbst für mich tun - Patientenkompetenz in der modernen Medizin. Zürich, Rüffer \& Rub, 2005.

18 Kübler-Ross E: Interviews mit Sterbenden. München, Droemer Knaur, 2001.

19 Zorn F: Mars. Frankfurt/M., Fischer, 2013.
20 Haushofer M: Die Wand. Frankfurt/M., Büchergilde Gutenberg, 2010.

21 Terzani T: Noch eine Runde auf dem Karussell. München, Droemer, 2014.

22 Mercier P: Nachtzug nach Lissabon. München, Carl Hanser, 2017.

23 Mehne S: Licht ohne Schatten. München, Heyne, 2014.

24 Mehne S: Der grosse Abflug. Ostfildern, Patmos, 2016.

25 Moorjani A: Heilung im Licht. München, Goldmann, 2015.

26 Haas H: Salutogenese und achtsamkeitsorientierte anthroposophische Psychotherapie am Beispiel ambulanter Gruppentherapien bei „Nervosität“. Schweiz Z Ganzheitsmed 2017; 29:DOI: 10.1159/000484462.

27 www.achtsamwerden.ch.

28 Haas H (Hrsg): Steiner R: Sich selbst erziehen Das Geheimnis der Gesundheit, ed 2. Dornach, Rudolf Steiner, 2014.

29 Kabat Zinn J: Zur Besinnung kommen: Die Weisheit der Sinne und der Sinn der Achtsamkeit in einer aus den Fugen geratenen Welt, ed 5. Freiburg i.Br., Arbor, 2013.

30 Domin H: Sämtliche Gedichte. Frankfurt/M. Fischer, 2013. 